\title{
EXISTENCE THEOREMS FOR OPTIMAL CONTROL OF QUASILINEAR PARABOLIC PARTIAL DIFFERENTIAL EQUATIONS
}

\author{
S. NABABAN and E. S. NOUSSAIR
}

(Received 24 May 1978)

(Revised 21 September 1978)

\begin{abstract}
The question on existence of optimal controls for a system governed by quasilinear parabolic partial differential equations which is linear in the control variables is considered. It is shown that whenever the controls converge in the weak * topology of $L^{\infty}$, the corresponding solutions converge uniformly. Using this result and results on lower semi-continuity of integral functionals, existence theorems for optimal controls are proved.
\end{abstract}

\section{Introduction}

In this paper, we shall consider the existence of optimal controls for an optimal control problem in which a given cost functional will be minimized subject to a system governed by the following quasilinear parabolic partial differential equations with first boundary conditions

$$
\begin{aligned}
\mathscr{L}_{u} \phi(u)(x, t) & =0, & & (x, t) \in Q=\Omega \times(0, T), \\
\phi(u)(x, t) & =\psi(x, t) & & \text { on } \Gamma,
\end{aligned}
$$

where $\Omega$ is an open bounded connected subset of $R^{n}$;

$$
\Gamma=\{(x, 0): x \in \Omega\} \bigcup \partial \Omega \times[0, T] ;
$$

$\partial \Omega$ denotes the boundary of $\Omega ; u \in D, D$ is the set of admissible controls to be defined later; $\psi$ is given function defined on $\bar{Q}$ and for each $u \in D$, the operator 
$\mathscr{L}_{u}$ is defined by

$$
\mathscr{L}_{u} \phi=\phi_{t}-\sum_{i, j=1}^{n} \frac{\partial}{\partial x_{j}}\left(a_{i j}(x, t, \phi) \phi_{x_{i}}\right)+a(x, t, \phi) u(x, t), \quad(x, t) \in \bar{Q}
$$

in which $a=\left(a_{1}, \ldots, a_{m}\right), u=\left(u_{1}, \ldots, u_{m}\right)$ and $a u=\sum_{i=1}^{m} a_{i} u_{i}$.

Related control problems to the problem considered in this paper can be found in $[1,4,7,8,9,10]$. In $[4,7,8,10]$, the authors considered systems of ordinary differential equations. In [1], Berkovitz considered an abstract control problem with $L^{p}$-bounded controls $(p \geqslant 1)$. However, the class of problems considered in [1] does not include the problem considered here. Indeed, in this paper the existence of solutions for the system is considered and the control restraint set depends also on the solution of the system, while in [1] the existence of solutions is not considered and the control restraint does not depend on the solution of the system. In [9], Noussair et al. considered a similar control problem to ours in which the existence of optimal controls was established using Filippov's implicit function lemma [5]. Our results are complementary to that given in [9] and overlap with the results in [1]. Further, our results can be considered as an extension of results in $[4,7,8,10]$ for ordinary differential systems to a quasilinear parabolic partial differential system.

\section{Notations}

Let $R^{n}$ denote the $n$-dimensional Euclidean space. For any $x \in R^{n}$, let $|x|=\left(\sum_{i=1}^{n}\left|x_{i}\right|^{2}\right)^{ \pm}$. The abbreviation a.e. means almost everywhere with respect to Lebesgue measure; $|E|$ denotes the Lebesgue measure of the measure set $E \subset R^{s}(s \geqslant 1), \partial B$ denotes the boundary of the set $B$ and $\bar{B}$ its closure.

A function $f: X \times Y \rightarrow R^{n}$ is said to be a Carathéodory function if $f(., t)$ is measurable on $X$ for every $t \in Y$ and $f(s,$.$) is continuous on Y$ for almost all $s \in X$.

Let $G$ denote a bounded connected subset of $R^{n}$; and denote by $C^{\prime}(G)(1 \leqslant l<\infty)$, the class of all $l$-times continuously differentiable real-valued functions on $G$. Also $C_{0}^{l}(G)$ denotes the class of all functions from $C^{l}(G)$ with compact support in $G$.

Let $L^{q}\left(G ; R^{m}\right)(m, q \geqslant 1)$ be the Banach space of all measurable functions $z: G \rightarrow R^{m}$ that are $q$ th-power integrable on $G$. Its norm is defined by

$$
\begin{array}{ll}
\|z\|_{q, G}=\left\{\int_{G}|z(x)|^{q} d x\right\}^{1 / q} & \text { for } 1 \leqslant q<\infty, \\
\|z\|_{\infty, G}=\underset{x \in G}{\operatorname{ess} \sup _{x \in G}|z(x)|} & \text { for } q=\infty .
\end{array}
$$


Let $L^{q, r}\left(G \times I ; R^{m}\right)(m, q, r \geqslant 1)$ be the Banach space of all measurable functions $z: G \times I \rightarrow R^{m}$ such that $\|z\|_{q, r, G}<\infty$, where

$$
\begin{aligned}
& \|z\|_{q, r, G \times I}=\left\{\int_{I}\left(\int_{Q}|z(x, t)|^{q} d x\right)^{r / q} d t\right\}^{1 / r}, \quad 1 \leqslant q, r<\infty, \\
& \|z\|_{a, \infty, G \times I}=\underset{t \in I}{\operatorname{ess} \sup \|z(., t)\|_{q, G}, \quad 1 \leqslant q<\infty, \quad r=\infty,} \\
& \|z\|_{\infty, r, G \times I}=\left\{\int_{I}\left(\|z(., t)\|_{\infty, G}\right)^{r} d t\right\}^{1 / r}, \quad q=\infty, \quad 1 \leqslant r<\infty,
\end{aligned}
$$

and

$$
\|z\|_{\infty, \infty, G \times I}=\underset{(x, t) \in G \times I}{\operatorname{ess} \sup }|z(x, t)| \text { for } q=r=\infty .
$$

The space $L^{q, q}\left(G \times I ; R^{m}\right)$ will be denoted by $L^{q}\left(G \times I ; R^{m}\right)$ and the norm $\|\cdot\|_{q, q, G \times I}$ by $\|.\|_{q, G \times I^{*}}$ Also $L^{q}\left(G ; R^{1}\right)\left(L^{q, r}\left(G \times I ; R^{1}\right)\right.$, respectively) will be denoted by $L^{q}(G)\left(L^{q, r}(G \times I)\right)$.

We define $W_{q}^{2: 1}(G \times I), 1 \leqslant q<\infty$, to be the Banach space of all functions $z$ from $L^{q}(G \times I)$ having generalized derivatives of the form $\left(\partial^{r} / \partial t^{r}\right)\left(\partial^{8} / \partial x^{8}\right)$ with any $r$ and $s$ satisfying the inequality $2 r+s \leqslant 2$. The norm in it is defined by

$$
\|z\|_{q, G \times I}^{(2)}=\|z\|_{q, G \times I}+\left\|\frac{\partial z}{\partial t}\right\|_{q, G \times I}+\left\|z_{x}\right\|_{q, G \times I}+\left\|z_{x x}\right\|_{q, G \times I} .
$$

Let $H^{\lambda}(G), 0<\lambda<1$, be the Banach space of all functions $z$ that are continuous in $G$ and have a finite norm

$$
|z|_{\alpha}^{(\lambda)}=\sup _{x, x^{\prime} \in G} \frac{\left|z(x)-z\left(x^{\prime}\right)\right|}{\left|x-x^{\prime}\right|^{\lambda}}+\max _{x \in \bar{G}}|z(x)|
$$

and let $H^{\lambda, \lambda / 2}(\overline{G \times I}), 0<\lambda<1$, be the Banach space of all functions $z$ that are continuous in $\overline{G \times I}$ and have a finite norm

$$
|z|_{G \times I}^{(\lambda)}=\|z\|_{x, G \times I}^{(\lambda)}+\|z\|_{l, G \times I}^{(\lambda / 2)}+\frac{\max }{\sigma \times I}|z|
$$

where

$$
\begin{aligned}
\|z\|_{x, G \times I}^{(\lambda)} & =\sup _{(x, t),\left(x^{\prime}, t\right) \in G \times I} \frac{\left|z(x, t)-z\left(x^{\prime}, t\right)\right|}{\left|x-x^{\prime}\right|^{\lambda}}, \\
\|z\|_{(, G \times I}^{(\lambda / 2)} & =\sup _{\left.(x, \lambda),(x,)^{\prime}\right) \in G \times I} \frac{\left|z(x, t)-z\left(x, t^{\prime}\right)\right|}{\left|t-t^{\prime}\right|^{\lambda / 2}} .
\end{aligned}
$$

Finally, let $H^{\lambda, \lambda / 2}(Q)$ be the set of all functions belonging to $H^{\lambda, \lambda / 2}\left(\bar{Q}_{1}\right)$ for any closed subdomain $\bar{Q}_{1} \subset Q$. 


\section{Preliminaries}

In this section, an existence theorem for solutions is given in Theorem 3.1. Further, it will be shown that whenever the controls converge in the weak * topology of $L^{\infty}\left(Q ; R^{m}\right)$ then the corresponding solutions converge uniformly on $\bar{Q}$. This result is presented in Lemma 3.3.

Let $\Omega$ be an open bounded connected subset of $R^{n}$. Let $T>0$ be a fixed constant and let $Q=\Omega \times(0, T)$ and $\Gamma=\{(x, 0): x \in \Omega\} \cup \partial \Omega \times[0, T]$.

Let $U_{K}$ denote that set of all bounded measurable functions $u: \bar{Q} \rightarrow R^{m}$ such that $|u(x, t)| \leqslant K$ for all $(x, t) \in \bar{Q}$. In the following $K>0$ denotes a positive fixed constant.

The following conditions will be referred to collectively as conditions $\left(B_{1}\right)$ :

(i) The functions $a_{i j}(x, t, \phi), i, j=1, \ldots, n$, and $a_{i}(x, t, \phi), i=1, \ldots, n$, are defined on $\bar{Q} \times R^{1}$ and take finite values for any finite $\phi$, and $(x, t) \in \bar{Q}$;

(ii) For all $(x, t) \in \bar{Q}$ and arbitrary $\phi \in R^{1}, \sum_{i, j=1}^{n} a_{i j}(x, t, \phi) \xi_{i} \xi_{j} \geqslant 0$ for all $\xi \in R^{n}$;

(iii) There exist non-negative constants $b_{1}$ and $b_{2}$ such that

$$
|\phi||a(x, t ; \phi)| \leqslant b_{1} \phi^{2}+b_{2}
$$

for all $(x, t) \in \bar{Q}$; and

(iv) $\psi \in H^{\beta}(\bar{Q})$ for some $\beta, 0<\beta<1$.

Let $M_{K}$ denote the positive constant defined by

$$
M_{K}=\inf _{\lambda>b_{1} K}\left(e^{\lambda x} \max _{\Gamma}\left\{|\psi|, \sqrt{\frac{b_{2} K}{\lambda-b_{1} K}}\right\}\right)
$$

DEFINITION 3.1. The boundary $\partial \Omega$ of $\Omega$ is said to satisfy condition $(A)$ if there exist constants $\alpha_{0}, \theta_{0}>0$ such that the inequality

$$
\left|\Omega_{\rho}^{i}\right| \leqslant\left(1-\theta_{0}\right)\left|B_{\rho}\right|
$$

holds for any ball $B_{\rho}$ with centre on $\partial \Omega$ of radius $\rho \leqslant \alpha_{0}$ and any connected component $\Omega_{\rho}^{i}$ of $\Omega_{\rho}$, where $\Omega_{\rho}=B_{\rho} \cap \Omega$.

The following conditions will be referred to as conditions $\left(B_{2}\right)$ : For $(x, t) \in \bar{Q}$ and $|\phi| \leqslant M_{K}$,

(i) The functions $a_{i j}(x, t, \phi), i, j=1,2, \ldots, n$, are continuous in $\phi$ and differentiable with respect to $x$ and $\phi$; and the function $a(x, t, \phi)$ is continuous in $\phi$;

$$
\nu|\xi|^{2} \leqslant \sum_{i, j=1}^{n} a_{i j}(x, t, \phi) \xi_{i} \xi_{j} \leqslant \mu|\xi|^{2}
$$


for all $\xi \in R^{n}$ and some constant $\nu, \mu>0$;

$$
\begin{aligned}
\left|a_{i j}(x, t, \phi)\right|+\left|\frac{\partial a_{i j}(x, t, \phi)}{\partial \phi}\right| & \leqslant \mu_{2}, \\
\left|\frac{\partial a_{i j}(x, t, \phi)}{\partial x_{i}}\right| & \leqslant \psi_{2}(x, t), \\
|a[x, t, \phi)| & \leqslant \psi_{3}(x, t),
\end{aligned}
$$

in which $\left\|\psi_{2}\right\|_{4 q, 4 r, Q} \leqslant \sqrt{ } \mu_{1},\left\|\psi_{3}\right\|_{2 q, 2 r, Q} \leqslant \mu_{1}$ for some constants $\mu_{1}, \mu_{2}>0$ with

$$
\frac{1}{r}+\frac{n}{2 q}=1-\chi_{1}
$$

where

$$
q \in\left[\frac{n}{2\left(1-\chi_{1}\right)}, \infty\right], \quad r \in\left[\frac{1}{1-\chi_{1}}, \infty\right], \quad 0<\chi_{1}<1
$$

for $n \geqslant 2$,

$$
q \in[1, \infty], r \in\left[\frac{1}{1-\chi_{1}}, \frac{2}{1-2 \chi_{1}}\right], \quad 0<\chi_{1}<\frac{1}{2}
$$

for $n=1$; and

(iii) $\partial \Omega$ satisfies the condition (A).

The existence of solutions for system (1.1) follows from Theorem 3.2 of Noussair et al. [9]. This theorem is quoted below without proof.

THEOREM 3.2. Let the assumptions $\left(B_{1}\right)$ and $\left(B_{2}\right)$ be satisfied. Then for each $u \in U_{K}$, the system (1.1) has a solution $\phi^{u} \in H^{\alpha, \alpha / 2}(\bar{Q}) \cap W_{2}^{2,1}\left(Q^{\prime}\right)$ with $\phi_{x}^{u} \in H^{\gamma, \gamma / 2}(Q)$, where $Q^{\prime}$ is any interior subdomain of $Q$ that is separated from $\Gamma$ by a distance $d>0$, and $0<\gamma, \alpha<1$ are constants determined by $K, n, \nu, \mu, \mu_{1}, \mu_{2}, q, M_{K}$ and $r$ from condition $B_{2}(i i)$. Furthermore, there exist positive constants $c$ and $c(d)$ such that for all $u \in U_{K}$, and for any subdomain $Q^{\prime}$ separated from $\Gamma$ by a distance $d>0$, the following estimates are valid

and

$$
\left|\phi^{u}\right|_{Q}^{(\alpha)} \leqslant c
$$

$$
\left|\phi_{x}^{u}\right|_{Q^{\prime}}^{(y)}+\left\|\phi_{l}^{u}\right\|_{2, Q^{\prime}}+\left\|\phi_{x x}^{u}\right\|_{2, Q^{\prime}} \leqslant c(d),
$$

where the constants $c$ and $c(d)$ are determined only by $n, M_{K}, \nu, \mu_{1}, \mu_{2}, \mu, q, r, K$, and the distance $d$.

LEMMA 3.3. Let the hypotheses of Theorem 3.2 be satisfied. Let $\left\{u_{n}\right\}$ be a sequence in $U_{K}$ such that $u_{n} \rightarrow u_{0}$ in the weak ${ }^{*}$ topology of $L^{\infty}\left(Q ; R^{m}\right)$. Let $\left\{\phi^{n}\right\}$ denote the 
sequence of solutions of system (1.1) corresponding to $u_{n}$. Then there exist a subsequence of $\left\{\phi^{n}\right\}$ which we shall denote by the original sequence and a function $\phi^{0} \in H^{\alpha, \alpha / 2}(\bar{Q})$ such that

(i) $\phi^{n} \rightarrow \phi^{0}$ uniformly on $\bar{Q}$,

(ii) $\phi^{0}$ is a solution of system (1.1) corresponding to $u_{0}$.

Proor. It follows from Theorem 3.2 that for any subdomain $Q^{\prime}$ separated from $\Gamma$ by a distance $d>0$,

$$
\begin{gathered}
\left|\phi^{n}\right|_{Q}^{(\alpha)} \leqslant c, \\
\left|\phi_{x}^{n}\right|(\gamma)+\left\|\phi_{Q^{\prime}}^{n}\right\|_{2, Q^{\prime}}+\left\|\phi_{x x}^{n}\right\|_{2, Q^{\prime}} \leqslant c(d),
\end{gathered}
$$

where the constants $c$ and $c(d)$ depend only on $n, M_{K}, \mu, \mu_{1}, \mu_{2}, \nu, q, r$ and $d$.

By using the diagonalization process given in [9], it can be easily shown that there exist a function $\phi^{0} \in H^{\alpha, \alpha / 2}(\bar{Q}) \cap W_{2}^{2,1}\left(Q^{\prime}\right)$ with $\phi_{x}^{0} \in H^{\gamma, \gamma / 2}(Q)$ and a subsequence of $\left\{\phi^{n}\right\}$ such that

$$
\left.\begin{array}{c}
\phi^{n} \rightarrow \phi^{0} \text { uniformly on } \bar{Q}, \\
\phi_{x}^{n} \rightarrow \phi_{x}^{0} \text { uniformly on } Q^{\prime}, \\
\phi_{i}^{n} \rightarrow \phi_{l}^{0} \text { weakly in } L^{2}\left(Q^{\prime}\right), \\
\phi_{x x}^{n} \rightarrow \phi_{x x}^{0} \text { weakly in } L^{2}\left(Q^{\prime}\right),
\end{array}\right\}
$$

where $Q^{\prime}$ is any closed subdomain of $Q$.

Let us first show that there exists a subsequence of $\left\{\left(\phi^{n}, u_{n}\right)\right\}$ which will be denoted by the original sequence such that

$$
\iint_{Q} a\left(x, t, \phi^{n}(x, t)\right) \cdot u_{n}(x, t) \cdot z(x, t) d x d t \rightarrow \iint_{Q} a\left(x, t, \phi^{0}(x, t)\right) \cdot u_{0}(x, t) \cdot z(x, t) d x d t
$$

for any $z \in L^{2}(Q)$. Since $\phi^{n} \rightarrow \phi^{0}$ uniformly in $\bar{Q}$, it is clear that $\left(\phi^{n}, u_{n}\right)-\left(\phi^{0}, u_{n}\right) \rightarrow 0$ in measure in $Q$. Further, $\left(\phi^{n}, u_{n}\right)$ and $\left(\phi^{0}, u_{n}\right)$ are bounded in the norm of the space $L^{\sigma_{1}}(Q) \times L^{\sigma_{2}}(Q), \sigma_{1}, \sigma_{2} \geqslant 1$. Hence, it follows from Lemma 3.1 of Berkovitz $[1$, p. 524] that

$$
\left(a\left(x, t, \phi^{n}(x, t)\right)-a\left(x, t, \phi^{0}(x, t)\right)\right) \cdot u_{n}(x, t) \rightarrow 0
$$

in measure in $Q$. Therefore, there exists a subsequence of $\left\{\left(\phi^{n}, u_{n}\right)\right\}$ such that

$$
\Psi^{n}(x, t)=\left(a\left(x, t, \phi^{n}(x, t)\right)-a\left(x, t, \phi^{0}(x, t)\right)\right) \cdot u_{n}(x, t) \rightarrow 0
$$

a.e. in $Q$. From the condition $B_{2}$ (ii), it follows that for $(x, t) \in \bar{Q}$,

$$
\left|\Psi^{n}(x, t)\right| \leqslant 2 K \psi_{3}(x, t),
$$


where $K$ is the bound on $u_{n}$ and $\psi_{3} \in L^{\mathbf{1}}(Q)$. Thus, it follows from the Lebesgue dominated convergence theorem that

$$
\lim _{n \rightarrow \infty} \iint_{Q} \Psi^{n}(x, t) \cdot z(x, t) d x d t=0 .
$$

Since $u_{n} \rightarrow u_{0}$ in the weak * topology of $L^{\infty}\left(Q ; R^{m}\right)$, it follows that

$$
\lim _{n \rightarrow \infty} \iint_{Q} a\left(x, t, \phi^{0}(x, t)\right) \cdot z(x, t) \cdot\left(u_{n}(x, t)-u_{0}(x, t)\right) d x d t=0 .
$$

Hence, (3.7) follows from (3.9) and (3.10). Furthermore, by virtue of (3.6) and (3.7) it is easy to see that $\phi^{0}$ is a solution of the system (1.1) corresponding to $u_{0}$. This completes the proof.

\section{Existence of optimal controls}

In this section, we consider the problem of existence of an optimal control. The exact formulation of the problem is given below and existence proofs in Theorems 4.1 and 4.3.

Let $B_{K}$ denote the $m$-dimensional sphere in $R^{m}$ with diameter $K$, where $K$ is a fixed positive constant. For each $(x, t, \psi) \in \bar{Q} \times R^{1}$, let $U(x, t, \psi)$ be a non-empty subset of $B_{K}$.

A bounded measurable function $u: Q \rightarrow B_{K}$ is called an admissible control if

(i) there exists a solution $\phi^{u}$ of system (1.1) corresponding to $u$; and

(ii) $u(x, t) \in U\left(x, t, \phi^{u}(x, t)\right)$ a.e. in $Q$.

Let $D$ denote the set of all admissible controls, and let $\Delta$ denote the set of all pairs ( $\left.\phi^{u}, u\right)$, where $u \in D$ and $\phi^{u}$ is a corresponding solution of system (1.1).

Let the cost functional $J$ be defined on $\Delta$ by

$$
J\left[\phi^{u}, u\right]=\iint_{Q} g\left(x, t, \phi^{u}(x, t), u(x, t)\right) d x d t,
$$

where $g(t, x, \psi, u)$ is a real-valued function on $Q \times R^{1} \times B_{K}$ and satisfies the following conditions which will be referred to as conditions $(H)$ :

(i) $g(x, t, \psi, u)$ is continuous in $(\psi, u)$ for a.e. $(x, t) \in Q$, measurable in $(x, t)$ for each $(\psi, u) \in R^{1} \times B_{K}$ and convex in $u$ for each $(x, t, \psi) \in Q \times R^{1}$; and

(ii) there exists a function $z \in L^{1}(Q)$ such that

$$
g(x, t, \psi, u) \geqslant z(x, t)
$$

for a.e. $(x, t) \in Q$ and for all $(\psi, u) \in R^{1} \times B_{K}$. 
We now state our control problem " $P$ ": subject to system (1.1), find an admissible pair $\left(\phi^{u_{0}}, u_{0}\right) \in \Delta$ such that

$$
J\left[\phi^{u_{0}}, u_{0}\right] \leqslant J\left[\phi^{u}, u\right]
$$

for all $\left(\phi^{u}, u\right) \in \Delta$. Such an admissible pair will be called an optimal pair.

THEOREM 4.1. Consider the problem P. Suppose that the following assumptions are satisfied.

(i) The conditions $\left(B_{1}\right),\left(B_{2}\right)$ and $(H)$ hold;

(ii) for each $(x, t, \psi) \in \bar{Q} \times R^{1}, U(x, t, \psi)$ is closed and convex; and

(iii) the mapping $U$ is upper semicontinuous with respect to inclusion (u.s.c.i.) on $\bar{Q} \times R^{1}$.

Then there exists an optimal pair $\left(\phi^{u_{0}}, u_{0}\right) \in \Delta$ for the problem $P$.

ProOF. From condition H(ii), it follows that $\inf \left\{J\left[\phi^{u}, u\right]:\left(\phi^{u}, u\right) \in \Delta\right\}=\sigma>-\infty$. Let $\left\{\left(\phi^{u_{k}}, u_{k}\right)\right\}_{k=1}^{\infty}$ be a minimizing sequence such that

$$
\lim _{k \rightarrow \infty} J\left[\phi^{u_{k}}, u_{k}\right]=\sigma .
$$

Since $u_{k}(x, t) \in B_{K}, k=1,2, \ldots$, there exist a subsequence of $\left\{u_{k}\right\}$ and a function $u_{0} \in L^{\infty}\left(Q ; R^{m}\right)$ such that

$$
u_{k} \rightarrow u_{0} \text { in the weak * topology of } L^{\infty}\left(Q ; R^{m}\right) .
$$

By Lemma 3.3, there exists a solution $\phi^{u_{0}}$ of system (1.1) corresponding to $u_{0}$ and a subsequence $\left\{\phi^{u_{k}}\right\}$ such that

$$
\phi^{u_{k} \rightarrow \phi^{u_{0}}} \text { uniformly in } \bar{Q} \text {. }
$$

We show that $u_{0}(x, t) \in U\left(x, t, \phi^{u_{0}}(x, t)\right)$ a.e. in $Q$. For this, let

$$
Q_{1}^{k}=\left\{(x, t) \in Q: u_{k}(x, t) \notin U\left(x, t, \phi^{u_{k}}(x, t)\right)\right\} .
$$

Let $Q_{1}=\bigcup_{k=1}^{\infty} Q_{1}^{k}$. Clearly $\left|Q_{1}\right|=0$. Let $Q_{0}=Q \backslash Q_{1}$. Let $\left(x_{0}, t_{0}\right) \in \bar{Q}_{0}$ be any regular point of the function $u_{0}$. We first show that

$$
u_{0}\left(x_{0}, t_{0}\right) \in U\left(x_{0}, t_{0}, \phi^{u_{0}}\left(x_{0}, t_{0}\right)\right) .
$$

Since $U$ is u.s.c.i., it follows that for a given $\varepsilon>0$, there exists a $\delta=\delta(\varepsilon)>0$ such that

$$
U(x, t, \phi) \subset U^{e}\left(x_{0}, t_{0}, \phi^{u_{0}}\left(x_{0}, t_{0}\right)\right)
$$

whenever $\left|(x, t)-\left(x_{0}, t_{0}\right)\right|<\delta$ and $\left|\phi-\phi^{u_{0}}\left(x_{0}, t_{0}\right)\right|<\delta$, where $U^{e}$ denotes the closed $\varepsilon$-neighborhood of $U$.

D 
From (4.5), it follows that there exists a positive integer $k_{0}$ such that for all $k>k_{0}$ and $(x, t) \in \bar{Q}$,

$$
\left|\phi^{u_{k}}(x, t)-\phi^{\mu_{0}}(x, t)\right|<\delta / 2 .
$$

Further, since $\phi^{u_{0}}$ is continuous on $\bar{Q}$, there exists $a \delta_{1}>0$ such that

$$
\left|\phi^{u_{0}}(x, t)-\phi^{u_{0}}\left(x_{0}, t_{0}\right)\right|<\delta / 2
$$

whenever $\left|(x, t)-\left(x_{0}, t_{0}\right)\right|<\delta_{1}$.

Let $\delta_{2}=\min \delta, \delta_{1}$ ). Then, it follows from (4.7), (4.8) and (4.9) that for all $k>k_{0}$

$$
U\left(x, t, \phi^{u_{k}}(x, t)\right) \subset U^{\epsilon}\left(x_{0}, t_{0}, \phi^{u_{0}}\left(x_{0}, t_{0}\right)\right)
$$

whenever $\left|(x, t)-\left(x_{0}, t_{0}\right)\right|<\delta_{2}$.

Let $E$ be any measurable subset of $Q$ such that $\left(x_{0}, t_{0}\right) \in E$ and $|E|<\delta_{2}$. Then, since $U^{\epsilon}(t, x, \phi)$ is convex and closed, it follows from (4.10) that for all $k>k_{0}$,

$$
\frac{1}{|E|} \iint_{E} u_{k}(x, t) d x d t \in U^{\epsilon}\left(x_{0}, t_{0}, \phi^{u_{0}}\left(x_{0}, t_{0}\right)\right)
$$

Since $U^{\epsilon}(x, t, \phi)$ is closed, it follows from (4.4) that

$$
\frac{1}{|E|} \iint_{E} u_{0}(x, t) d x d t \in U^{\epsilon}\left(x_{0}, t_{0}, \phi^{\mu_{0}}\left(x_{0}, t_{0}\right)\right),
$$

and consequently,

$$
u_{0}\left(x_{0}, t_{0}\right)=\lim _{|E| \rightarrow 0}\left(\frac{1}{|E|} \iint_{E} u_{0}(x, t) d x d t\right) \in U^{e}\left(x_{0}, t_{0}, \phi^{u_{0}}\left(x_{0}, t_{0}\right)\right) .
$$

Since $\varepsilon$ was arbitrary and $U\left(x_{0}, t_{0}, \phi^{u_{0}}\left(x_{0}, t_{0}\right)\right)$ is closed, it follows that (4.6) holds. Further, since almost every point in $Q$ is a regular point of $u_{0}$, we obtain that

$$
u_{0}(x, t) \in U\left(x, t, \phi^{u_{0}}(x, t)\right) \text { a.e. in } Q .
$$

Hence, $\left(\phi^{u_{0}}, u_{0}\right) \in \Delta$. Further, it follows from (4.4), $\mathrm{t}(4.5)$, assumptions $(\mathrm{H})$ and Theorem 1 of $[2$, p. 53] that

$$
\liminf _{k \rightarrow \infty} J\left[\phi^{u_{k}}, u_{k}\right] \geqslant J\left[\phi^{u_{0}}, u_{0}\right]
$$

Therefore, it follows from (4.3) and (4.11) that

$$
J\left[\phi^{u_{0}}, u_{0}\right]=\sigma,
$$

That is $\left(\phi^{u_{0}}, u_{0}\right)$ is an optimal pair. This completes the proof. 
REMARK 4.2. Theorem 4.1 remains valid when the conditions $(H)$ on $g$ is replaced by the weaker conditions $\left(\mathrm{H}^{\prime}\right)(6, \mathrm{p} .524]$ :

(i) $g(x, t, \psi, u)$ is lower semicontinuous in $(\psi, u)$ and convex in $u$ for each $(x, t, \psi) \in Q \times R^{1} ;$ and

(ii) there exist functions $\gamma, \lambda \in L^{1}(Q)$ such that for all $\psi \in R^{1}, u \in B_{K}$ and $(x, t) \in Q$,

$$
g(x, t, \psi, u) \geqslant \gamma(x, t)|u|+\lambda(x, t) \text {. }
$$

We now consider the case when the cost integrand $g$ and the control restraint set $U$ satisfy the following conditions which will be referred to as conditions $(G)$ :

(i) $g(x, t, \psi, u)$ is continuous in all its variables and convex in $u$ for each $(x, t, \psi) \in Q \times R^{1}$

(ii) for each $(x, t, u) \in Q \times B_{K}$ and $\psi, \psi^{\prime} \in R^{1}$,

$$
\left|g(x, t, \psi, u)-g\left(x, t, \psi^{\prime}, u\right)\right| \leqslant \beta\left(\left|\psi-\psi^{\prime}\right|\right)
$$

where $\beta$ is a non-negative function defined on $[0, \infty)$ satisfying

$$
\lim _{\delta \rightarrow 0^{+}} \beta(\delta)=0
$$

(iii) $U$ is independent of $\psi$ and for each $(x, t) \in \bar{Q}, U(x, t)$ is closed and convex.

THEOREM 4.3. Consider the problem $P$. Suppose that the conditions $\left(B_{1}\right),\left(B_{2}\right)$ and (G) hold. Then, there exists an optimal pair $\left(\phi^{u_{0}}, u_{0}\right) \in \Delta$ for the problem $P$.

ProOf. From estimate (3.2) and the condition $G(i)$, we can easily show that $\inf \left\{J\left[\phi^{u}\right]:\left(\phi^{u}, u\right) \in \Delta\right\}=\sigma_{0}>-\infty$. As in the proof of Theorem 4.1, let $\left\{\left(\phi^{u_{k}}, u_{k}\right)\right\}$, $u_{0}$ and $\phi^{u_{0}}$ be, respectively, a minimizing sequence, a function in $L^{\infty}\left(Q ; R^{m}\right)$ and a solution of system (1.1) corresponding to $u_{0}$ such that

$$
\lim _{k \rightarrow \infty} J\left[\phi^{u_{k}}, u_{k}\right]=\sigma_{0},
$$

and

$$
u_{k} \rightarrow u_{0} \text { in the weak * topology of } L^{\infty}\left(Q ; R^{m}\right) \text {; }
$$

$$
\phi^{u_{k}} \rightarrow \phi^{u_{0}} \text { uniformly on } \bar{Q}
$$

Using condition G(iii) and an argument similar to that given in the proof of Theorem 4.1, we can easily show that $\left(\phi^{u_{0}}, u_{0}\right) \in \Delta$. 
From (4.1), it follows that

$$
\begin{aligned}
J\left[\phi^{u_{k}}, u_{k}\right] & =\iint_{Q} g\left(x, t, \phi^{u_{k}}(x, t), u_{k}(x, t)\right) d x d t \\
& =\iint_{Q}\left(g\left(x, t, \phi^{u_{k}}(x, t), u_{k}(x, t)\right)-g\left(x, t, \phi^{u_{0}}(x, t), u_{k}(x, t)\right)\right) d x d t \\
& +\iint_{Q} g\left(x, t, \phi^{u_{0}}(x, t), u_{k}(x, t)\right) d x d t .
\end{aligned}
$$

Using the condition $G(i i)$, it follows from (4.14) that

$$
\lim _{k \rightarrow \infty} \iint_{Q}\left(g\left(x, t, \phi^{u_{k}}(x, t), u_{k}(x, t)\right)-g\left(x, t, \phi^{u_{0}}(x, t), u_{k}(x, t)\right)\right) d x d t=0 .
$$

Since $g$ is continuous in its all variables and convex in $u$, it follows from (4.13) and the theorem of $[3$, p. 213] that

$\liminf _{t \rightarrow \infty} \iint_{Q} g\left(x, t, \phi^{u_{0}}(x, t), u_{k}(x, t)\right) d x d t \geqslant \iint_{Q} g\left(x, t, \phi^{u_{0}}(x, t), u_{0}(x, t)\right) d x d t$

Hence, it follows from (4.15), (4.16) and (4.17) that

$$
\liminf _{k \rightarrow \infty} J\left[\phi^{u_{k}}, u_{k}\right] \geqslant J\left[\phi^{u_{0}}, u_{0}\right]
$$

Therefore, $J\left[\phi^{u_{0}}, u_{0}\right]=\sigma_{0}$, that is $\left(\phi^{u_{0}}, u_{0}\right)$ is an optimal pair. This completes the proof.

\section{References}

[1] L. D. Berkovitz, "A lower closure theorem for abstract control problems with $L_{p}$-bounded controls", J. Optimization Theory \& Appl. 14 (1974), 521-528.

[2] L. D. Berkovitz, "Lower semicontinuity of integral functionals", Trans. Amer. Math. Soc. 192 (1974), 51-57.

[3] W. H. Fleming, "Optimal control of partially observable diffusions", SIAM J. Control 6 (1968), 194-214.

[4] J. P. Gossez, "Existence of optimal controls for some non-linear processes", J. optimization Theory \& Appl. 3 (1969), 89-97.

[5] C. J. Himmelberg, M. Q. Jacobs and F. S. Van Vleck, "Measurable multifunctions, selectors and Filippov's implicit function lemma", J. Math. Anal. Appl. 25 (1969), 276-289.

[6] A. D. Ioffe, "On lower semicontinuity of integral functions I", SIAM J. Control \& Optim. 18 (1977), 521-538. 
[7] M. Q. Jacobs, "Some existence theorems for linear optimal control problems", SIAM J. Control 5 (1967), 418-437.

[8] E. B. Lee and L. Markus, "Optimal control for nonlinear processes", Arch. Rat. Mech. Anal. 8 (1961), 36-58.

[9] E. S. Noussair, S. Nababan and K. L. Teo, "On the existence of optimal controls for quasilinear parabolic partial differential equations", submitted for publication.

[10] M. Vidyasagar, "On the existence of optimal controls", J. Optimization Theory \& Appl. 17 (1975), 273-278.

School of Mathematics

University of N.S.W.

P.O. Box 1

Kensington, N.S.W. 2033 